\title{
Delta Scuti Stars: Selected Recent Highlights
}

\author{
G. Handler \\ South African Astronomical Observatory, P.O. Box 9, Observatory \\ 7935, South Africa
}

\begin{abstract}
An overview of recent progress in the research on $\delta$ Scuti stars is given. Some intriguing results and open problems are pointed out, and some ideas for future investigations are provided.
\end{abstract}

\section{Introduction}

It is almost not necessary to write a review about the progress in the field of $\delta$ Scuti and related stars these days. A very successful workshop on these objects has been held in Vienna the week before this meeting, and almost everything one ever wanted to know about these stars can be found in the proceedings of that workshop (Breger \& Montgomery 2000).

Nevertheless, I will attempt to give an overview of recent interesting results here, focusing on topics less emphasised at the Vienna meeting, but still comprehensive enough that the reader can obtain a picture of what is going on in the field. For possibly less experienced readers interested in $\delta$ Scuti stars emphasis has been placed on providing a good number of references.

\section{High-Amplitude $\delta$ Scuti Stars (HADS)}

Large-scale surveys definitely have had the largest impact on this sub-field of $\delta$ Scuti star research. OGLE (see Morgan, Simet, \& Bargenquast 1998) and MACHO data (Alcock et al. 1999) have been successful in the discovery and analysis of HADS.

Examination of the Fourier parameters of HADS light curves is predicted to aid in determining the radial overtone number of the pulsations (e.g. Bono et al. 1997), which in turn allows the determination of distances to HADS in the Bulge, or even in extragalactic systems (Poretti 1999). However, observations do not show such a clear picture as the theoretical predictions; the reasons for this are not well understood. Another interesting problem is posed by $\delta$ Scuti stars with anomalous light curve shapes (e.g. Rodríguez et al. 1997), whose nature is also still in question. Poretti (2000) provides more detailed discussions.

Some HADS have potential to calibrate mode-identification tools and pulsational model calculations for $\delta$ Scuti stars. These are double-mode pulsators with additional nonradial modes excited (e.g. Walraven, Walraven, \& Balona 1992; Garrido \& Rodríguez 1996; Zhou et al. 1999), whose radial modes are gauges to which the additional modes must be fitted. Radial triple-mode pulsators (Fitch \& Szeidl 1976; Antipin 1997; Handler, Pikall, \& Diethelm 1998a) will help to 
check the validity of post-main sequence pulsation models, e.g. following Kovács \& Buchler (1994).

\section{Pre-Main Sequence $\delta$ Scuti Stars}

There are two major motivations to study these stars in detail. First, constraints on the inner structure of these objects can be obtained and pre-main sequence evolutionary models can be tested (Marconi \& Palla 1998). Second, these stars are expected to show large evolutionary period changes, and they may be the only objects among $\delta$ Scuti stars where evolutionary changes can actually be measured; for (post) main-sequence objects period changes are predominantly due to other (presently poorly understood) effects (Breger \& Pamyatnykh 1998).

Only a few pre-main sequence $\delta$ Scuti stars have been found so far (Breger 1972; Kurtz \& Marang 1995), and the evolutionary state for some of them is not unambiguous (Peña et al. 2000). However, Marconi et al. (2000) report the discovery of five more related objects, and initial astrophysical results are to be expected soon.

\section{Multimode Nonradially Pulsating $\delta$ Sct Stars \& Asteroseismology}

\subsection{Multisite Observations and Pulsational Mode Identification}

This has been the most active branch of research on $\delta$ Scuti stars in recent years. To illustrate this, Table 1 summarises the major multisite campaigns carried out between late 1997 and 1998. This table only comprises campaigns yielding about $200 \mathrm{hr}$ of measurement - sometimes significantly more!

Table 1. Recent major multisite campaigns on $\delta$ Scuti stars

\begin{tabular}{ll}
\hline Target(s) & Representative reference(s) \\
\hline V647 Tau & Liu et al. (2000) \\
XX Pyx & Arentoft et al. (2000) \\
BV and BN Cnc & Frandsen et al. (2000) \\
BN Cnc & Dall (2000) \\
29 Cyg & Mkrtichian et al. (2000) \\
V534 Tau & Fox et al. (2000) \\
BI CMi & Breger et al., in preparation \\
38 Eri & Paparó et al., in preparation \\
\hline
\end{tabular}

A number of multisite observations has triggered detailed model calculations. Pamyatnykh et al. (1998) performed a direct comparison of the observed frequency spectrum of XX Pyx with (unstable) model frequencies, attempting to obtain the closest match between the two. However, they could not find a unique best-fitting model, suggesting that theory is not accurate enough yet to reproduce the observationally determined frequencies within their measurement accuracy. This may partly be due to an insufficient treatment of rotation. 
Soufi, Goupil, \& Dziembowski (1998) developed a third-order perturbation theory which can help to resolve some of the discrepancies. Pérez Hernández et al. (1999) computed the effects of rotation on the photometric magnitudes of models. This needs to be corrected for when determining the basic parameters of a star from photometry.

A different approach to asteroseismology was made by Hernández et al. (1998), who assumed two radial modes each to be excited in four $\delta$ Scuti stars in Praesepe. On this basis they illustrated the potential of simultaneous modelling of the frequency spectra to obtain constraints on the stars themselves, but also on the whole cluster. Michel et al. (1999) extended this method by performing stability analysis, and showed this can provide constraints on the amount of surface convection.

Despite these large theoretical and observational efforts, the main obstacle for asteroseismology of $\delta$ Scuti stars still remains: the lack of reliable mode identifications for many of the observed pulsation frequencies.

An improved photometric method for mode identification was proposed and tested by Balona \& Evers (1999). They developed a statistical technique which takes into account variability information in several filters (an extension of the common two-filter method, e.g. Garrido, Garcia-Lobo, \& Rodríguez 1990), and they also demonstrated problems for the photometric method caused by surface convection.

Another very prominent technique uses of the ratio of equivalent width amplitudes of Balmer lines and of photometric amplitudes (Bedding et al. 1996). Balona \& Dziembowski (1999) examined this method and found it sensitive to the same effects as the pure photometric approach. Hence they were not surprised by the agreement between these two strategies (Breger et al. 1999a).

Further difficulties for mode identification can arise from mode coupling (Pamyatnykh et al. 1998; Breger et al. 1999a). This means that mode frequencies can be modified and that the surface appearance of the coupled modes can no longer be represented by a simple spherical harmonic. For instance, a radial mode coupled to a quadrupole mode could actually appear to be dipole mode in diagnostics for mode identification! Fortunately, this does not affect a large number of modes, but the problem becomes more severe the denser the mode spectrum is and the faster the star rotates.

The conclusion implied by all these difficulties is: mode-identification methods should be used, since they can provide important guidelines. However, they should be cross-calibrated and their validity must be verified, but the results should not be trusted blindly.

\subsection{Nonlinearities}

Nonlinear behaviour of $\delta$ Scuti stars has been noted in a number of studies. However, physical interpretations of these effects have rarely been attempted. Therefore, I find it useful to point out some of the findings in the hope or triggering some closer investigations.

It is well-known that $\delta$ Scuti stars can show frequency variability over long time scales (e.g. Breger 2000). It has also frequently been noted that after removing all detected periodic signals from the light curves of these stars, the residuals show larger scatter than the observational accuracy would predict. 


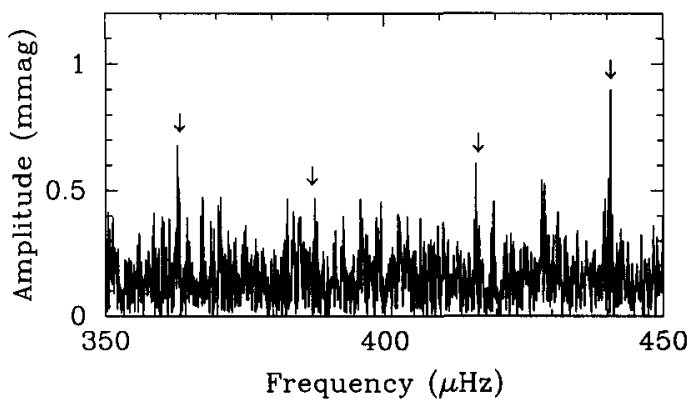

Figure 1. Amplitude spectrum of XX Pyx after removing an optimised synthetic light curve consisting of all of the pulsation frequencies detected so far in the data. The location of the four strongest modes is indicated; peaks very close to them can be found.

Could there be a connection? As an example, the amplitude spectrum of the recent campaign on XX Pyx (see Arentoft et al. 2000), after removal of a number of detected pulsation frequencies, is shown in Fig. 1.

In this figure, signals with frequencies very close to that of the four strongest modes excited in the star can be seen. This suggests that amplitude and/or frequency variations (with a time scale of about 20 days) of at least some of the modes occurred during the observations: $\delta$ Scuti stars can change the appearance of their mode spectrum on much shorter time scales than previously thought! The time scales of these variations are consistent with inverse growth rates for the modes in models for the star. This can be taken as evidence that resonant coupling between the modes is the reason for these temporal changes (Handler et al. 1998b). This is an interesting result. However, there is one snag: the theory of resonant mode coupling can explain such observations nicely, but it cannot provide predictions on which its validity can be tested.

In any case, one should also keep in mind that similar effects are seen in other classes of pulsating stars, for instance white dwarfs (e.g. see Bond et al. 1996; Kleinman et al. 1998), and there is, of course, the famous Blazhko effect in RR Lyrae stars (e.g. Kurtz et al. 2000; Kolenberg et al. 2000). Could it be that the same physics operates in different variable star groups?

The second nonlinear effect clearly present in $\delta$ Scuti star pulsations manifests itself in linear combination frequencies in the amplitude spectra. For the low-amplitude multimode pulsators, these have first been discovered for XX Pyx by Handler et al. (1996), who showed that they originate from distortion of the light curve caused by the stellar material being unable to respond fully elastically to the acceleration induced by pulsation; they are not independent modes excited by resonance (Dziembowski 1982).

In this respect it is regrettable that such linear combination frequencies are often called "nonlinear coupling terms" in the literature. This is confusing, since it can let one think these signals are due to resonant mode coupling. This has never been proven. I suggest that this designation be dropped whenever inexact. 


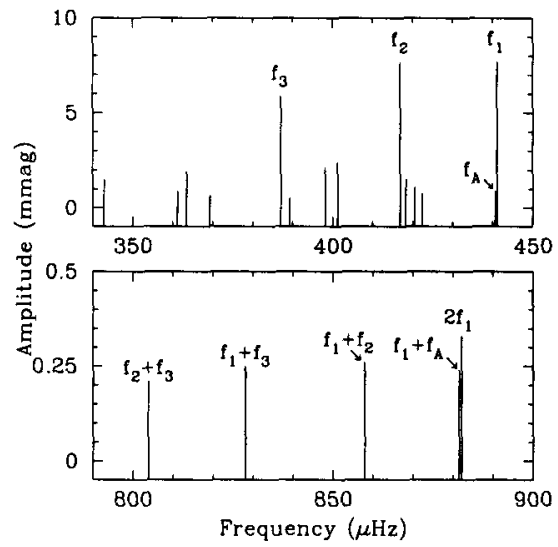

Figure 2. Schematic amplitude spectra for XX Pyx as observed in the 1998 multisite campaign. The three strongest modes as well as their linear combinations are indicated. The peak labelled $f_{A}$ is one of the very close companions suggesting amplitude or frequency variability.

Can we learn some physics from the linear combination frequencies? Yes. For pulsating DA white dwarfs model calculations suggest that linear combination frequencies originate in the surface convection zone (Brickhill 1992; Wu $1997,2000)$. This is also conceivable for $\delta$ Scuti stars since they possess surface convection zones as well. Maybe the linear combination frequencies originate in the driving zone itself ( $\mathrm{Wu}$, private communication). However, detailed model calculations examining the origin of linear combination frequencies for $\delta$ Scuti stars are not yet available.

Linear combination frequencies may also be able to provide clues on the intrinsic nature of suspected pulsation frequencies. Fig. 2 shows a part of the schematic amplitude spectrum of XX Pyx for the region where the pulsations are excited and for the linear combination frequency domain. As can be seen, the sum frequencies of the three strongest modes are all present together with one 2f-harmonic. However, the linear combination of $f_{1}$ and its close companion (labelled as $f_{A}$ ) is also present, suggesting that $f_{A}$ does not indicate amplitude or frequency variability of $f_{1}$, but corresponds to an independent pulsation mode.

\section{Conclusion}

Research on $\delta$ Scuti stars is flourishing. Large observational and theoretical studies are, and have been made. Despite (or because of!) this activity there is still much to be done.

\section{References}

Alcock, C., et al. (the MACHO collaboration) 1999, AJ, submitted 
Antipin, S. 1997, A\&A, 326, L1

Arentoft, T., Handler, G., Shobbrook, R. R., et al. 2000, in these proceedings, p. 469

Balona, L. A. \& Evers, E. A. 1999, MNRAS, 302, 349

Balona, L. A. \& Dziembowski, W. A. 1999, MNRAS, in press

Bedding, T. R., Kjeldsen, H., Reetz, J., \& Barbuy, B. 1996, MNRAS, 280, 1155

Bond, H. E., Kawaler, S. D., Ciardullo, R., et al. 1996, AJ, 112, 2699

Bono, G., Caputo, F., Cassisi, S., et al. 1997, ApJ, 477, 346

Breger, M. 1972, ApJ, 171, 539

Breger, M. 2000, in these proceedings, p. 421

Breger, M. \& Pamyatnykh, A. A. 1998, A\&A, 332, 958

Breger, M. \& Montgomery, M. H. (eds.) 2000, ASP Conf. Ser., Delta Scuti and Related Stars, (San Francisco: ASP), in press

Breger, M., Pamyatnykh, A. A., Pikall, H., \& Garrido, R. 1999a, A\&A, 341, 151

Breger, M., Handler, G., Garrido, R., et al. 1999b, A\&A, 349, 225

Brickhill, A. J. 1992, MNRAS, 259, 519

Dziembowski, W. A. 1982, Acta Astron., 32, 147

Fitch, W. S. \& Szeidl, B. 1976, ApJ, 203, 616

Fox Machado, L., Li, Z. P., Michel, E., et al. 2000, in these proceedings, p. 477

Frandsen, S., Pigulski, A., and the STACC collaboration 2000, in these proceedings, p. 473

Garrido, R. \& Rodríguez, E. 1996, MNRAS, 281, 696

Garrido, R., Garcia-Lobo, E., \& Rodríguez, E. 1990, A\&A, 234, 262

Handler, G., Breger, M., Sullivan, D. J., et al. 1996, A\&A, 307, 529

Handler, G., Pikall, H., \& Diethelm, R. 1998a, IBVS, No. 4549

Handler, G., Pamyatnykh, A. A., Sullivan, D. J., Audard, N., \& Nitta, A. 1998b, MNRAS, 295,377

Hernández, M. M., Pérez Hernández, F., Michel, E., et al. 1998, A\&A, 338, 511

Kleinman, S. J., Nather, R. E., Winget, D. E., et al. 1998, ApJ, 495, 424

Kolenberg, K., Aerts, C., Chadid, M., \& Gillet, D. 2000, in these proceedings, p. 286

Kovács, G. \& Buchler, J. R. 1994, A\&A, 281, 749

Kurtz, D. W. \& Marang, F. 1995, MNRAS, 276, 191

Kurtz, D. W., Alcock, C., Allsman, R. A., et. al. 2000, in these proceedings, p. 291

Liu, Y. Y., Michel, E., Garrido, R., et al. 2000, in these proceedings, p. 481

Marconi, M. \& Palla, F. 1998, ApJ, 507, L141

Marconi, M., Ripepi, V., Alcalà, J., et al. 2000, in these proceedings, p. 459

Michel, E., Hernández, M. M., Houdek, G., et al. 1999, A\&A, 342, 153

Mkrtichian, D., Kusakin, A., Koval, V., et al. 2000, in these proceedings, p. 494

Morgan, S. M., Simet, M., \& Bargenquast, S. 1998, Acta Astron., 48, 509 
Pamyatnykh, A. A., Dziembowski, W. A., Handler, G., \& Pikall, H. 1998, A\&A, 333,141

Peña, J. H., Paparó, M., Peniche, R., et al. 2000, in these proceedings, p. 467

Pérez Hernández, F., Claret, A., Hernández, M. M., \& Michel, E. 1999, A\&A, 346,586

Poretti, E. 1999, A\&A, 343, 385

Poretti, E. 2000, in these proceedings, p. 83

Rodríguez, E., González-Bedolla, S. F., Rolland, A., et al. 1997, A\&A, 328, 235

Soufi, F., Goupil, M.-J., \& Dziembowski, W. A. 1998, A\&A, 334, 911

Walraven, Th., Walraven, J., \& Balona, L. A. 1992, MNRAS, 254, 59

$\mathrm{Wu}$, Y. 1997, PhD thesis, California Institute of Technology

$\mathrm{Wu}, \mathrm{Y} .2000$, in these proceedings, p. 508

Zhou, A.-Y., Rodríguez, E., Jiang, S.-Y., Rolland, A., \& Costa, V. 1998, MNRAS, 308, 631

\section{Discussion}

Giuseppe Bono: Did you find any correlation between nonlinear effects and the position of these pulsators inside the instability strip?

Gerald Handler: For the nonradially pulsating stars we have too few well-studied objects to make any meaningful statement to help answering your question.

S. Oliveira Kepler: You did not comment that the leftover power in the Fourier Transform might be due to bad extinction correction and the difference in amplitude with wavelength due to different heights of the observatories.

Gerald Handler: We always see residual mounds of power in the amplitude spectra, i.e. the noise is apparently enhanced in the frequency region where the pulsations occur, but it is smaller towards both higher and lower frequencies. Hence, bad extinction corrections (which would only introduce an $1 / \mathrm{f}$ component in the Fourier Transforms) cannot be the cause for the leftover power. Besides, we always take great care in accounting for extinction and sky transparency variations properly!

Concerning your second suspicion, I made calculations estimating the size of spurious effects due to pass-band mismatches between different observatories involved in multisite campaigns (Handler 1998, Baltic Astronomy 7, 227). I found no effects large enough to disturb frequency analyses for typical observations of low-amplitude $\delta$ Scuti pulsators. However, it may become dangerous when examining high-amplitude variables for weak additional signals.

Don Kurtz: The $f_{1}+f_{A}$ combination frequency is expected, if $f_{A}$ describes amplitude or phase variation of $f_{\mathbf{1}}$.

Gerald Handler: I agree. However, the amplitude ratios of $f_{1} / f_{A}$ and $2 f_{1} /\left(f_{1}+f_{A}\right)$ should then be the same. This is clearly not the case! 